\title{
SrRietveld: A program for automating Rietveld refinements for high throughput powder diffraction studies
}

\author{
P. Tian ${ }^{a, b}$ W. Zhou ${ }^{b}$ J. Liu,${ }^{b}$ Y. Shang,${ }^{b}$ C. L. FArrow,${ }^{b}$ P. JuhÁs ${ }^{b}$ And \\ S. J. L. BiLlinge ${ }^{b, c *}$ \\ ${ }^{a}$ Department of Physics and Astronomy, Michigan State University East Lansing, \\ Michigan, 48824, USA, ${ }^{b}$ Department of Applied Physics and Applied Mathematics, \\ Columbia University New York, New York, 10027, USA, and ${ }^{c}$ Condensed Matter \\ Physics and Materials Science Department, Brookhaven National Laboratory, Upton, \\ New York, 11973, USA. E-mail: sb2896@columbia.edu \\ (Received 0 XXXXXXX 0000; accepted 0 XXXXXXX 0000)
}

\begin{abstract}
SrRietveld is a highly automated software toolkit for Rietveld refinement. Compared to traditional refinement programs, it is more efficient to use and easier to learn. It is designed for modern high throughput diffractometers and capable of processing large numbers of data-sets with minimal effort. The new software currently uses conventional Rietveld refinement engines, automating GSAS and FullProf refinements. However, as well as automating and extending many tasks associated with these programs, it is designed in a flexible and extensible way so that in the future these engines can be replaced with new refinement engines as they become available. SrRietveld is an open source software package developed in Python.
\end{abstract}




\section{Introduction}

The Rietveld refinement method is widely used for determining crystalline material structures from x-ray or neutron diffraction data (Rietveld, 1969; Rietveld, 1967). In this method, a theoretical line profile is calculated from a structure model that is refined using a least-squares approach until it matches the discrete data from neutron or x-ray powder diffractometers. Many computer programs have been developed for Rietveld refinement (Larson \& Von Dreele, 2004; Rodriguez-Carvajal, 1993; Murray et al., 1990; Howard \& Hunter, 1998; Izumi, 1989; Bergmann et al., 1998; Coelho, 2004). However, most software programs of this type require intensive user inputs and there is also a sharp learning curve for new users. The high data throughput from new generation diffractometers (Proffen et al., 2002; Williams et al., 1998; Mason et al., 2006; Hodges \& Crawford, 2000), such as POWGEN at the Spallation Neutron Source (SNS) at Oak Ridge National Laboratory, is more than the conventional Rietveld refinement software can easily handle. Poor convergence of current programs is another obstacle for accurate determination of refined parameters and conventional refinement software often diverges, requiring significant human intervention to find the optimal structure solution. SrRietveld is designed to improve the user experience in computer aided Rietveld refinement.

The new software makes use of the existing refinement programs GSAS (Larson \& Von Dreele, 2004) and FullProf (Rodriguez-Carvajal, 1993), which are already widely used. These programs are used by SrRietveld as refinement engines and SrRietveld provides an automation layer and a graphical user interface. The new functionalities are implemented in Python packages that can manipulate and communicate with the refinement engines. The refinement process can be controlled from SrRietveld and the results can be displayed and analyzed. This architecture separates the controlling scripts from the refinement engines and also allows the implementation of other IUCr macros version 2.1.1: 2009/05/11 
engines in the future. Using SrRietveld, average users can set up high-throughput refinement quickly and save time in analyzing large numbers of data-sets. Meanwhile, advanced users are still able to customize the refinement routines, create their own Python scripts, or even develop new software applications based on SrRietveld's functionalities. The SrRietveld codes are open source.

\section{SrRietveld}

\subsection{Design Principles}

SrRietveld has been developed as part of the NSF-funded distributed data analysis for neutron scattering measurements (DANSE) project in the Python language (Python, 1990) using object oriented programming (OOP) concepts. Python is extensively used in scientific software development. It is cross-platform and suitable for fast development. Also there are many well designed packages for scientific programming and visualization, such as Matplotlib (Matplotlib, 2010), NumPy (NumPy, 2005) and SciPy (SciPy, 2007). The OOP design enables SrRietveld to be easily maintained and extended.

Refinement information and results are stored in a flexible HDF5 data structure (HDF5, 2010) allowing easy organization of a large number of refinements. SrRietveld also provides powerful tools for visualizing refinements. Fits can be plotted in real-time during refinement, as can selections of refined parameters plotted against refinement number or some other meta-parameter such as temperature. Pre-written scripts are provided for automating common tasks, such as refining from coarse guesses of initial parameters, sequential refinement, etc. It is also possible for advanced users to develop their own macro scripts and contribute to the development of SrRietveld. Users are supported through an online community where features can be requested and bugs reported.

IUCr macros version 2.1.1: 2009/05/11 


\subsection{Implementation}

The architecture of SrRietveld is shown in Figure 1.

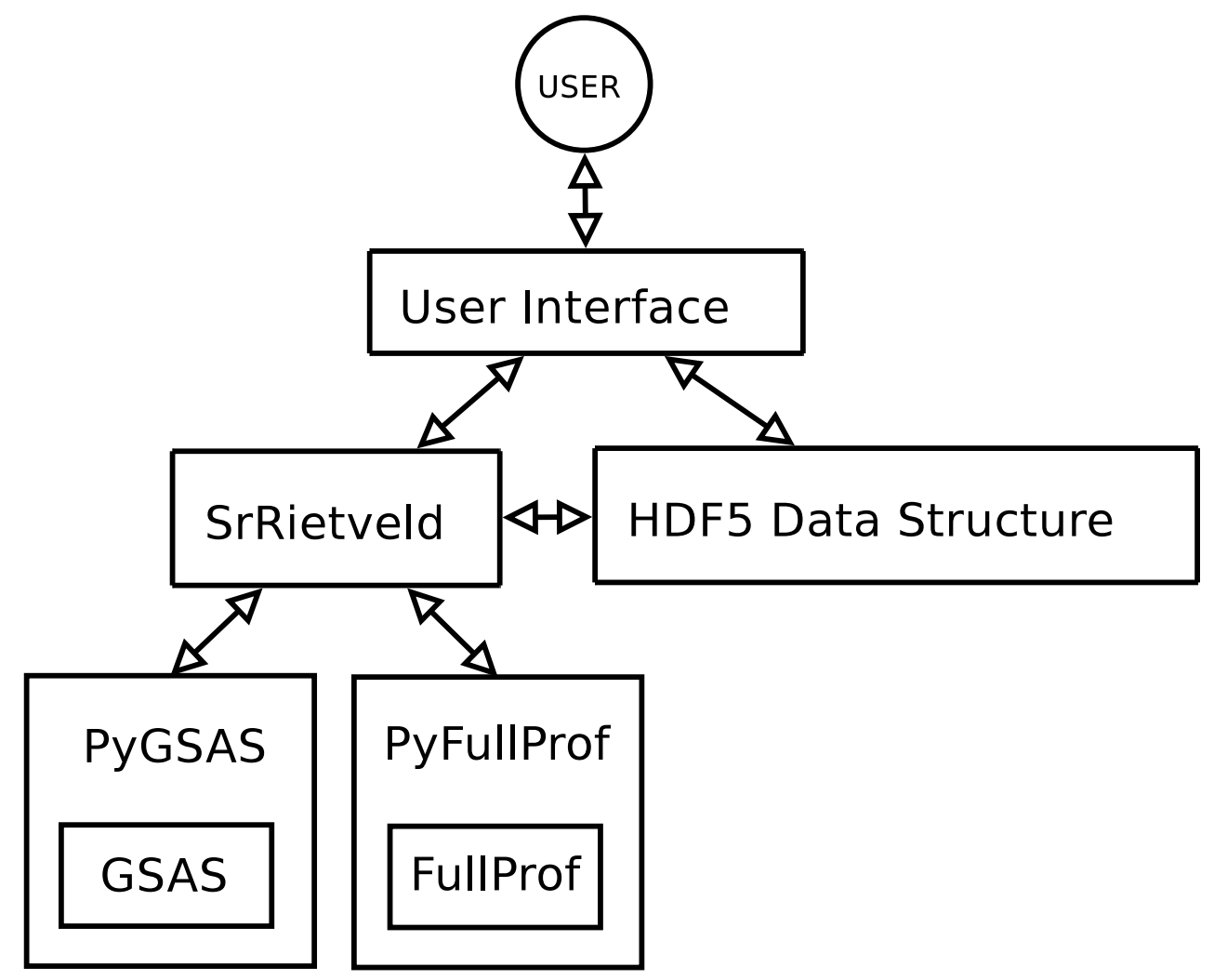

Fig. 1. SrRietveld software architecture. The User Interface, SrRietveld, HDF5 Data Structure, PyGSAS, and PyFullprof are python packages in the SrRietveld project. The GSAS and Fullprof represent the underlying refinement engines.

SrRietveld consists of several programmatic units, indicated by boxes in Figure 1. The SrRietveld user interface is a full-featured graphical user interface (GUI) that enables the user to conveniently configure the refinement routines, modify inputs, and investigate and analyze the results. The GUI interacts with a control layer that coordinates communication with the refinement engines, PyGSAS and PyFullProf. PyGSAS and PyFullProf are Python libraries that give programmatic access to GSAS and FullProf. The implementation of PyFullProf, PyGSAS and the control layer ensures that SrRietveld has consistent behavior even while using different engines, allowing refineIUCr macros version 2.1.1: 2009/05/11 
ments to be readily carried out on the same data using either engine. The HDF5 data structure has been designed to be relatively small in size and quick to load, even when handling thousands of datasets.

SrRietveld incorporates most of the major functionality supported by the current versions of FullProf and GSAS. However, some features, such as the refinement of magnetic phases, are not implemented, though this is planned for a later release.

The program captures errors and attempts to recover automatically when a refinement diverges, and thus increases the robustness of this sequential refinement process. Figure 2.2 shows a screen-shot of SrRietveld when it is carrying out sequential refinements on $\mathrm{NaCl}$ x-ray powder diffraction data collected as a function of temperature, as described in detail in the Figure caption.

IUCr macros version 2.1.1: 2009/05/11 


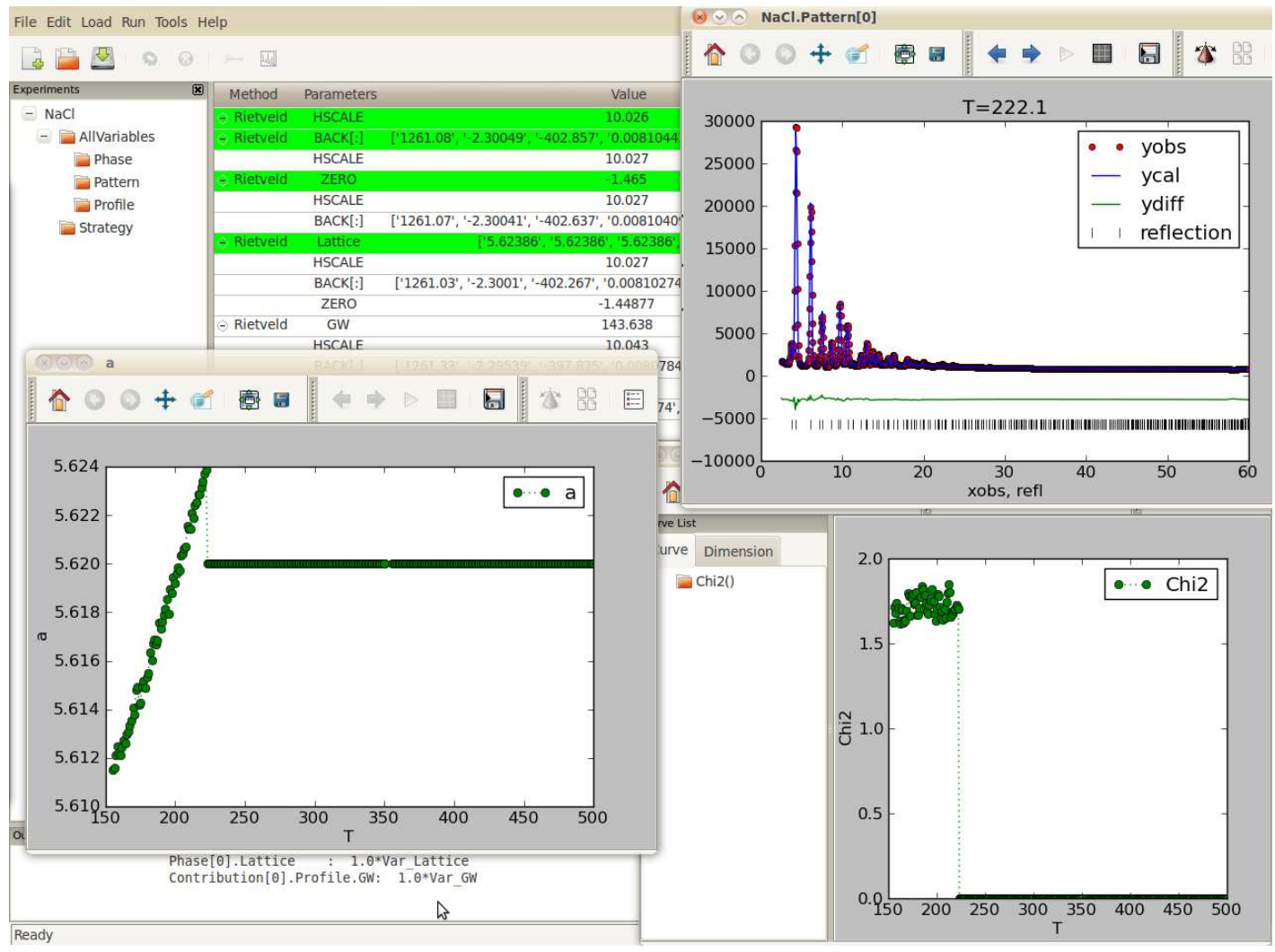

Fig. 2. Screen-shot of SrRietveld's plotting window during a sequential refinement. The data are $\mathrm{NaCl}$ which were measured as a function of temperature (see Section 3 for details). The top right panel shows the fit of the dataset being currently refined. The crosses are the data, the solid red line is the calculated pattern and the greenline offset below is the difference curve. The black tick-marks show the positions of Bragg peak reflections in the model. We have chosen to monitor the $a$-lattice parameter values (bottom left panel) refined from each of the data-sets. The $\chi^{2}$ of the fits are shown in the lower right panel, allowing issues to be addressed as they emerge. Text outputs from the program are displayed in a text window that is just evident in the lower left of the Figure.

In addition to the ease-of-use, SrRietveld provides flexibility and extensibility to advanced users. Since SrRietveld is modular and designed with focus on extensibility, the default behavior of the software can be redefined. Advanced user scripts can be written in Python to give new functionalities. Such a script is demonstrated below, which fits a Debye model for the lattice dynamics to the temperature dependence of 
the refined thermal parameters. In future releases we will allow easy incorporation of user-defined scripts into the program for easy sharing. Scientific programmers can also implement the application programming interfaces provided in SrRietveld into their own applications so SrRietveld functionality can be incorporated into other programs.

SrRietveld is open source software distributed under the BSD License (BSD, 1983). It is free to use, subject to the copyright restrictions and disclaimer, though we ask that papers published from work done using SrRietveld cite this paper, as well as the paper describing the particular refinement engine used (FullProf or GSAS). More information can be obtained from the project web pages (SrRietveld, 2009) or by contacting professor Simon Billinge (sb2896@columbia.edu).

\subsection{Future Developments}

SrRietveld is under active development and we encourage users to post bug reports and feature requests on the SrRietveld online community group (details in the documentation). The plans for future releases include support for parallel computation, magnetic diffraction, and features for engineering diffraction analysis.

\section{Example}

To demonstrate a typical use case, sequential refinements were carried out on x-ray powder diffraction patterns measured from $\mathrm{NaCl}$ at a series of temperatures. The data were collected at beamline 11-ID-B of the Advanced Photon Source (APS) at Argonne National Laboratory (ANL). The $\mathrm{NaCl}$ powder was obtained from Puratronic $\AA$ with $99.999 \%, 5 \mathrm{~N}$ grade purity. To demonstrate the ability to handle data from a high

throughput instrument, many data-sets were collected using a low-resolution high throughput mode (Chupas et al., 2003). The sample was contained in a $1 \mathrm{~mm}$ diameter kapton tube mounted perpendicular to the synchrotron beam and cooled using an

IUCr macros version 2.1.1: 2009/05/11 
Oxford cryostream liquid nitrogen cooler. Data were collected at 307 temperature points between $155 \mathrm{~K}$ and $500 \mathrm{~K}$ on a $2 \mathrm{D}$ Perkin-Elmer amorphous silicon detector mounted $128 \mathrm{~mm}$ behind the sample and perpendicular to the beam. The beam energy was $58.26 \mathrm{keV}$ giving an x-ray wavelength of $0.2128 \AA$. The temperature was ramped continuously at $3 \mathrm{~K} / \mathrm{min}$ during data collection.

To improve the convergence during the sequential refinement, the refined results from the previous temperature point were used as initial values in the refinement on the data set at the following temperature point. The first point to be refined was the lowest temperature point. A refinement scheme was used whereby parameters were turned on in turn according to normal practice (Young, 1993) beginning with a Lebail refinement (Le Bail et al., 1987) of the lattice parameters and the background, followed by Rietveld refinements where the scale parameter, zero shift parameters, lattice parameter, etc., were switched on in turn. This procedure is automated in SrRietveld and requires no user input after initial setup. Although in principle in a sequential refinement it should not be necessary to follow this scheme at every step since the initial parameters from the previous refinement are used and are already close to the minimum in practice we find that at some temperature points the refinement will still diverge if this scheme is not followed. However, SrRietveld provides great flexibility. For example, if speed of refinement is an issue the scheme could be changed so that all parameters are switched on at once after the first refinement, and the stepby-step scheme only turned on if a refinement diverges. Because SrRietveld controls the engines with scripts, there is great flexibility for automating such a procedure.

In the $\mathrm{NaCl}$ refinements, the full-set of refinable parameters used was the scale factor, the zero shift factor, the lattice parameter, the background parameters, the peak profile parameters, and the isotropic thermal displacement parameters. The starting model was taken from the literature (Wilson, 1995). Refinements were carried out

IUCr macros version 2.1.1: 2009/05/11 
on the same data using both GSAS and FullProf engines within SrRietveld. Both temperature series refinements used the same SrRietveld script, with the refinement engine changed from FullProf to GSAS by simply loading a different type of of template in the GUI. The ease of refining using both GSAS and FullProf engines is one of the great strengths of SrRietveld as we show here. Although GSAS and FullProf models are highly similar, there are subtle differences in how they handle experimental effects. For example, the way they handle the background function is quite different. In the FullProf example, user defined points are imported and a linear interpolation is applied between them for background correction. In the GSAS case, the background function is fitted to the data together with Rietveld profile. We have used the background function 6, a power series with negative power terms (Larson \& Von Dreele, 2004)). Also, the peak profiles are slightly different. The pseudo-Voigt peak profile function was used in the FullProf case. The GSAS refinements used profile function 3, which is also a pseudo-Voigt, but parametrized in a slightly different way (Larson \& Von Dreele, 2004). These model differences result in slight differences in the physical parameters refined from the data from each engine, as evident in Fig. 3 and Table 1. 


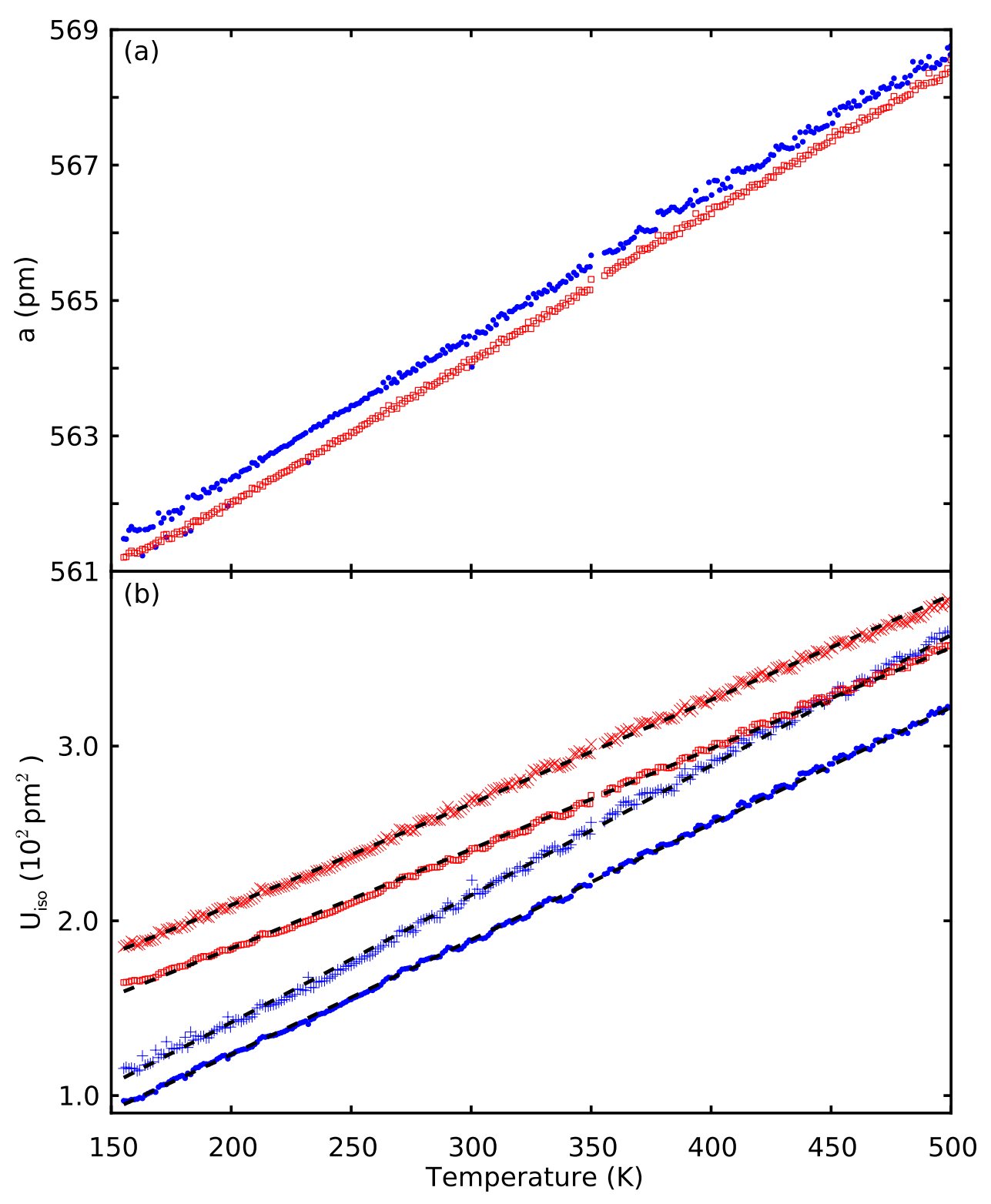

Fig. 3. Temperature dependence of SrRietveld refinement results for $\mathrm{NaCl}$ using FullProf (blue dots) and GSAS (red squares) engines. (a) lattice parameter, $a$, and (b) atomic displacement parameters, $U_{i s o}$, at $\mathrm{Na}$ (crosses for FullProf, plus markers for GSAS) and $\mathrm{Cl}$ (dots for FullProf, squares for GSAS) sites. Dashed lines mark fitted Debye model curves. 
Table 1. Rietveld refinement results for $\mathrm{NaCl}$ at room temperature.

\begin{tabular}{llrl}
\hline & $a(p m)$ & $U_{\text {iso }, \mathrm{Na}}\left(\mathrm{pm}^{2}\right)$ & \multicolumn{1}{c}{$U_{\text {iso } \mathrm{Cl}}\left(\mathrm{pm}^{2}\right)$} \\
\hline GSAS & $564.00(5)$ & $267.2(3)$ & $238.46(13)$ \\
FullProf & $564.35(9)$ & $214.4(6)$ & $187.3(3)$ \\
literature* & $563.9^{\dagger}$ & $22.9(4)$ & $189(4)$ \\
\hline
\end{tabular}

* (Butt et al., 1973) ${ }^{\dagger}$ measured at $295 \mathrm{~K}$

Another feature of SrRietveld is that the parameters from many refinements are retained in the Python data-structure, allowing post-processing scripts of arbitrary complexity to be written. Here this feature is illustrated by writing a script to fit the Debye model to the refined Debye-Waller factors from $\mathrm{Na}$ and $\mathrm{Cl}$ from each of the engines. It is possible for advanced users to make plug-ins for SrRietveld, which can be saved and reused by the user, and also readily shared with other users.

The theoretical values of $U_{i s o}$ are calculated from (Fischer et al., 1978)

$$
U_{i s o}(T)=\frac{1}{8 \pi^{2}}\left\{\frac{6 h^{2}}{M k_{B} \theta_{D}}\left[\frac{1}{4}+\left(\frac{T}{\theta_{D}}\right)^{2} \int_{0}^{\theta_{D} / T} \frac{x d x}{e^{x}-1}\right]\right\}+\sigma_{o}
$$

where the refinable parameters are $\theta_{D}$ and $\sigma_{o}$, the Debye temperature and offset, respectively. Additionally, $T$ is temperature in Kelvin, $M$ is the mass of the ion in question, $k_{B}$ and $h$ are the Boltzmann and Planck constants, respectively. The fitting to the refined $U_{\text {iso }}$ data is carried out with the least square optimization algorithm available in the SciPy Python scientific computing package (SciPy, 2007). The best-fit lines are shown in Fig. 3 as dashed lines through the data and the quantitative results from Debye fitting are listed in Table 2, where $\Theta_{D, N a}$ and $\Theta_{D, C l}$ represent the Debye temperatures from the fittings on $U_{i s o}$ values of $\mathrm{Na}$ and $\mathrm{Cl}$, respectively. 
Table 2. Debye temperatures of $\mathrm{NaCl}$ from fitting the Debye model to $U_{i s o}(T)$ values refined using different refinement engines. The numbers in brackets are the estimated standard deviation on the last digit.

\begin{tabular}{lrrrr}
\hline & $\Theta_{D, \mathrm{Na}}(K)$ & $\sigma_{o, \mathrm{Na}}\left(\mathrm{pm}^{2}\right)$ & $\Theta_{D, \mathrm{Cl}}(K)$ & $\sigma_{o, \mathrm{Cl}}\left(\mathrm{pm}^{2}\right)$ \\
\hline GSAS & $322.4(3)$ & $78.6(3)$ & $266.6(3)$ & $61.5(3)$ \\
FullProf & $289.2(4)$ & $-18.1(5)$ & $248.5(2)$ & $-16.9(2)$ \\
\hline
\end{tabular}

The differences between the Debye temperatures and offsets refined from each engine are much larger than the estimated standard deviations. The esds give a measure of the precision of the fit, and not the accuracy of the Debye temperatures. To understand this, note that the dashed lines fit well to the $U_{i s o}$ curves, but the slopes and offsets of the curves are clearly different. The differences in slopes and offsets come from differences in the mathematical model for the line-shapes and backgrounds used in FullProf and GSAS, respectively. This illustrates the advantage of being able to refine data using two refinement engines. If the FullProf and GSAS models are equally valid and we cannot say one or the other is inferior, then the dispersion of results from the two engines gives a realistic estimate of our uncertainty in the accuracy of the refined parameters. In this case we may take into account the result from both engines to get estimate of $\Theta_{D, \mathrm{Na}}=305 \pm 17 \mathrm{~K}$ and $\Theta_{D, \mathrm{Cl}}=257 \pm 9 \mathrm{~K}$ where the estimated uncertainties now reflect accuracy rather than precision. The corresponding offset parameters obtained by combining the Rietveld and GSAS results are $30 \pm 50 \AA^{-1}$ and $20 \pm 40 \AA^{-1}$ for $\mathrm{Na}$ and $\mathrm{Cl}$, respectively. Therefore there is no evidence for a static offset in the Debye model that would indicate a significant number of static defects in the structure.

In other experiments, the Debye temperature of $\mathrm{NaCl}$ can be determined either by measuring the elastic constant or by fitting the specific heat data. The $\Theta_{D}$ values measured with these two methods are $321.2 \pm 1.6 \mathrm{~K}$ and $320.6 \pm 1.5 \mathrm{~K}$, respectively, reported by Lewis et. al. (Lewis et al., 1967). Broadly speaking the Debye temperatures IUCr macros version 2.1.1: 2009/05/11 
measured here are in good agreement. Because of the simplicity of the Debye model, and the different way that different measurements weight the phonon density of states, the different approaches are not expected to yield the same Debye temperatures. Furthermore, the thermodynamic measurements give some average of the full density of states whereas the diffraction measurement differentiates the behavior of $\mathrm{Na}$ and $\mathrm{Cl}$. The agreement between the values determined from diffraction and those obtained from other methods are therefore quite acceptable.

\section{Acknowledgments}

The refinement engines used in the current release of SrRietveld are FullProf and GSAS. Along with many others in the community, we would like to thank Juan Rodriguez-Carvajal and the other FullProf developers and Robert B. Von Dreele, Brian H. Toby and the other GSAS developers, for their enormous efforts in developing FullProf and GSAS. We would also like to thank the other developers in the DANSE project for useful advice and help. We appreciate the cooperation and valuable suggestions from Emil Božin, Jason Hodges, Ashfia Huq, Ke An, Paolo Radaelli and Laurent Chapon. We thank Timur Dykhne for help with the documentation. In addition to their suggestions, Aziz Daoud-Aladine, Jon Hanson, Vanessa Peterson, and Andrew Studer shared data for testing which we are grateful for. The authors also would thank Christos Malliakas for providing the $\mathrm{NaCl}$ sample and assistance on the measurements on this sample. SrRietveld was developed as part of the DANSE (distributed data analysis of neutron scattering experiments) project, which is funded by the National Science Foundation under grant DMR-0520547. Use of the Advanced Photon Source was supported by the U.S. Department of Energy, Office of Science, Office of Basic Energy Sciences, under Contract No. W-31-109-Eng-38.

\section{References}

IUCr macros version 2.1.1: 2009/05/11 
Bergmann, J., Friedel, P. \& Kleeberg, R. (1998). Newsletter of the commission on Powder Diffraction of the International Union of Crystallography, 20, 5-8.

BSD, (1983). http://www.opensource.org/licenses/bsd-license.php.

Butt, N. M., Machonis, A. A. \& Cadoff, I. B. (1973). Acta Crystallogr. 29, 727-728.

Chupas, P. J., Qiu, X., Hanson, J. C., Lee, P. L., Grey, C. P. \& Billinge, S. J. L. (2003). J. Appl. Crystallogr. 36, 1342-1347.

Coelho, A. A. (2004). TOPAS General Profile and Structure Analysis Software for Powder Diffraction Data; http://members.optusnet.com.au/ alancoelho/.

Fischer, P., Sosnowska, I. \& Szymanski, M. (1978). J. of Phys. C: Solid State Phys. 11, $1043-1051$.

HDF5, (2010). http://www.hdfgroup.org/HDF5/.

Hodges, J. P. \& Crawford, R. K. (2000). Powgen3 - high-resolution general purpose powder diffractometer conceptual design study. Http://neutrons.ornl.gov/instrument_systems/beamline_11a_powgen/.

Howard, C. J. \& Hunter, B. A., (1998). A computer program for Rietveld analysis of x-ray and neutron powder diffraction patterns. Lucas Heights Research Laboratories, pp 1-27.

Izumi, F. (1989). The Rigaku Journal, 6, 10-20.

Larson, A. C. \& Von Dreele, R. B. (2004). General structure analysis system. Report No. LAUR-86-748, Los Alamos National Laboratory, Los Alamos, NM 87545.

Le Bail, A., Duroy, H. \& Fourquet, J. L. (1987). Mater. Res. Bull. 23(3), 447-452.

Lewis, J. T., Lehoczky, A. \& Briscoe, C. V. (1967). Phys. Rev. 161(3), 877-887.

Mason, T. E., Abernathy, D., Anderson, I., Ankner, J., Egami, T., Ehlers, G., Ekkebus, A., Granroth, G., Hagen, M., Herwig, K., Hodges, J., Hoffmann, C., Horak. C.. Horton, L., Klose, F., Larese, J., Mesecar, A., Myles, D., Neuefeind, J., Ohl, M., Tulk, C., Wang, X. \& Zhao, J. (2006). Physica B, 385-395, 955-960.

Matplotlib, (2010). http://matplotlib.sourceforge.net.

Murray, A. D., Cockcroft, J. K. \& Fitch, A. N. (1990). Powder diffraction program library $(P D P L)$. London: University College London.

NumPy, (2005). http://numpy.scipy.org.

Proffen, T., Egami, T., Billinge, S. J. L., Cheetham, A. K., Louca, D. \& Parise, J. B. (2002). Appl. Phys. A, 74, s163-s165.

Python, (1990). http://www.python.org.

Rietveld, H. M. (1967). Acta Crystallogr. 22, $151-152$.

Rietveld, H. M. (1969). J. Appl. Crystallogr. 2, 65-71.

Rodriguez-Carvajal, J. (1993). Physica B, 192, 55-69.

SciPy, (2007). http://www.scipy.org.

SrRietveld, (2009). http://www.diffpy.org/doc/srrietveld.

Williams, W. G., Ibberson, R. M., Day, P. \& Enderby, J. E. (1998). Physica B, 241-243, $234-236$.

Wilson, A. J. C. (ed.) (1995). International tables for crystallography, vol. C. Kluwer Academic Publishers.

Young, R. A. (1993). The Rietveld Method, vol. 5 of International Union of Crystallography Monographs on Crystallography. Oxford: Oxford University Press.

\section{Synopsis}

SrRietveld, a Python based Rietveld refinement program is described. SrRietveld extends and automates the popular existing Rietveld programs FullProf and GSAS to facilitate studies that involve many refinements such as the analysis of large quantities of data-sets. 